\title{
On the Use of Multiple Auxiliary Variables in Estimation of Current Population Mean in Two-Occasion Successive (Rotation) Sampling
}

\author{
G. N. Singh, J. P. Karna and S. Prasad \\ Indian School of Mines, Dhanbad, India
}

\begin{abstract}
The present work emphasizes the role of several stable auxiliary variables at both the occasions to improve the precision of estimates at current occasion in two-occasion successive sampling. A chain-type multiple linear regressions in ratio estimator has been proposed and its theoretical properties are examined. Relative comparison of efficiencies of the proposed estimator with the sample mean estimator, when there is no matching from the previous occasion and the natural successive sampling estimator, when no auxiliary information is used have been made. Theoretical results have been well supported with empirical illustrations.
\end{abstract}

Key words: Successive sampling, stable auxiliary variables, bias, mean square error, optimum replacement policy.

\section{Introduction}

Surveys often gets repeated on many occasions for estimating same characteristics at different points of time. The information collected on previous occasion may be used to study the change or the total value over occasion for the character and also in addition to study the average value for the most recent occasion. For example, in agricultural survey one may be interested in (i) estimating the average amount of yield per acre of an important crop (say wheat) in current season (ii) estimating the change in average amount of yield for a province (county) over two different seasons and (iii) estimating both parameters from (i) and (ii) simultaneously. The successive method of sampling consists of selecting sample units on different occasions such that some units are common with samples selected on previous occasions. If sampling on successive occasions is done according to a specific 
rule, with partial replacement of sampling units, it is known as successive sampling. A key issue is the extent to which elements sampled at a previous occasion should be retained in the sample selected at the current occasion; this is termed as optimum replacement policy.

The method of successive sampling was developed by Jessen [1] and extended by Patterson [2], Eckler [3], Rao and Graham [4], Sen [5, 6, 7], Cochran [8], Gupta [9], Singh et al. [10], Feng and Zou [11], Biradar and Singh [12], Singh and Singh [13], Singh [14], Singh [15], Singh and Priyanka [16, 17, 18, 19] and Singh and Karna [20, 21] among others.

Sometimes, information on several auxiliary variables may be readily available or may be made easily available by diverting a small amount of fund available for the survey. For instance, to study the case of public health and welfare of a state or country, several factors are available that can be treated as auxiliary variables, such as the number of beds, number of doctors and supporting staffs in different hospitals, the amount of funds available for medicine etc. may be known. Likewise, there may be several information available, which if efficiently utilized can improve the precision of estimates. Following the work of Singh and Priyanka [19], the objective of the present work is to propose estimator for estimating the population mean at current occasion using information on several stable auxiliary variables available on both the occasions. Utilizing information on $p$ stable auxiliary variables, chain-type multiple linear regressions in ratio estimator has been proposed. Relative comparison of efficiencies of the proposed estimator with the sample mean estimator, when there is no matching from the previous occasion and the natural successive sampling estimator, when no auxiliary information is used have been made. Empirical studies show the highly significant gains for the proposed estimator.

\section{Formulation of Estimator}

Let $U=\left(U_{1}, U_{2}, \ldots, U_{N}\right)$ be the finite population of $N$ units, which has been sampled over two occasions. The character under study is denoted by $x(y)$ on the first (second) occasion, respectively. It is assumed that the information on p-stable (non negative integer constant) auxiliary variables $z_{j}(j=1,2, \ldots, p)$ with known population means and correlated to $x$ and $y$ 
on the first and second occasions respectively, are readily available on the first as well as on the second occasion. A simple random sample (without replacement) of size $n$ is drawn on the first occasion. A random sub-sample of size $m=n \lambda$ is retained (matched) for its use on the second occasion, while a fresh simple random sample (without replacement) of size $u=(n-m)=$ $n \mu$ is drawn on the second occasion from the entire population so that the sample size on the second occasion is also $n . \quad \lambda$ and $\mu(\lambda+\mu=1)$ are the fractions of the matched and fresh samples, respectively, at the current(second) occasion. The values of $\lambda$ or $\mu$ should be chosen optimally. Following notations have been considered for further use:

$\bar{X}, \bar{Y}$ : Population means of $x$ and $y$ respectively.

$\bar{Z}_{j}$ : Population means of the $j^{t h}(j=1,2, \ldots, p)$ auxiliary variable.

$\bar{x}_{n}, \bar{x}_{m}, \bar{y}_{u}, \bar{y}_{m}$ : Sample means of the respective variables based on the sample sizes shown in suffices.

$\bar{z}_{j u}, \bar{z}_{j n}, \bar{z}_{j m}$ : Sample means of the $j^{t h}(j=1,2, \ldots, p)$ auxiliary variable based on the sample sizes shown in suffices.

$\rho_{y x}, \rho_{x z j}, \rho_{y z j}, \rho_{z j z k}$ : Correlation coefficients between the variables shown in suffices, where $j \neq k=1,2, \ldots, p$.

$S_{x}^{2}=(N-1)^{-1} \sum_{i=1}^{N}\left(x_{i}-\bar{X}\right)^{2}$ : Population mean square of $\mathrm{x}$. $S_{y}^{2}, S_{z j}^{2}$ : Population mean squares of y and $z_{j}(j=1,2, \ldots, p)$ respectively.

To estimate the population mean $\bar{Y}$ on the current (second) occasion, utilizing information on $p$-stable auxiliary variables, two different estimators are suggested. One is a simple multiple linear regression estimator based on sample of size $u=(n \mu)$ drawn afresh on the current occasion and is given by

$$
T_{1}=\bar{y}_{u}+\sum_{j=1}^{p} b_{y z j}(u)\left(\bar{Z}_{j}-\bar{z}_{j u}\right)
$$

The second estimator is a chain-type multiple linear regressions in ratio estimator based on the sample of size $m=(n \lambda)$ common with both the occasions and is defined as

$$
T_{2}=\frac{\bar{y}_{m}^{*}}{\bar{x}_{m}^{*}} \bar{x}_{n}^{*}
$$

where $\bar{y}_{m}^{*}=\bar{y}_{m}+\sum_{j=1}^{p} b_{y z j}(m)\left(\bar{Z}_{j}-\bar{z}_{j m}\right), \bar{x}_{m}^{*}=\bar{x}_{m}+\sum_{j=1}^{p} b_{x z j}(m)\left(\bar{Z}_{j}-\right.$ 
$\left.\bar{z}_{j m}\right), \bar{x}_{n}^{*}=\bar{x}_{n}+\sum_{j=1}^{p} b_{x z j}(n)\left(\bar{Z}_{j}-\bar{z}_{j n}\right)$ and $b_{y z j}(u), b_{y z j}(m), b_{x z j}(n)$ and $b_{x z j}(m)(j=1,2, \ldots, p)$ are the sample regression coefficients between the variables shown in suffices and based on the sample sizes shown in braces. Considering the convex linear combination of the estimators $T_{1}$ and $T_{2}$ defined in equations (1) and (2), we have the final estimator $T$ for estimating the population mean $\bar{Y}$ on the current (second) occasion, which is defined as:

$$
T=\varphi T_{1}+(1-\varphi) T_{2}
$$

where $\varphi$ is the unknown constant to be determined under certain criterion.

\section{Properties of the Estimator $T$}

Since, $T_{1}$ and $T_{2}$ are simple multiple linear regression and chain-type multiple linear regressions in ratio estimator, they are biased for population mean $\bar{Y}$, therefore, the resulting estimator $T$ defined in equation (3) is also biased estimator of $\bar{Y}$. The bias $B($.$) and mean square error M($.$) of the$ estimator $T$ up-to the first order of approximations are derived under large sample approximations using the following transformations:

$\bar{y}_{u}=\bar{Y}\left(1+e_{1}\right), \quad \bar{y}_{m}=\bar{Y}\left(1+e_{2}\right), \quad \bar{x}_{m}=\bar{X}\left(1+e_{3}\right), \quad \bar{x}_{n}=\bar{X}\left(1+e_{4}\right)$, $\bar{z}_{j u}=\bar{Z}_{j}\left(1+e_{5 j}\right), \quad \bar{z}_{j m}=\bar{Z}_{j}\left(1+e_{6 j}\right), \quad \bar{z}_{j n}=\bar{Z}_{j}\left(1+e_{7 j}\right), \quad s_{y z j}(u)=$ $S_{y z j}\left(1+e_{8 j}\right), \quad s_{z j}^{2}(u)=S_{z j}^{2}\left(1+e_{9 j}\right), \quad s_{y z j}(m)=S_{y z j}\left(1+e_{10 j}\right), \quad s_{z j}^{2}(m)=$ $S_{z j}^{2}\left(1+e_{11 j}\right), \quad s_{x z j}(m)=S_{x z j}\left(1+e_{12 j}\right), \quad s_{x z j}(n)=S_{x z j}\left(1+e_{13 j}\right)$ and $s_{z j}^{2}(n)=S_{z j}^{2}\left(1+e_{14 j}\right)$; Such that $E\left(e_{i}\right)=0$ and $E\left(e_{h j}\right)=0$, $\left|e_{i}\right| \leq 1 \quad \forall i=1,2,3,4$ and $\left|e_{h i}\right| \leq 1 \quad \forall h=5,6,7, \ldots, 14$. where $(j=1$, $2,3, \ldots, p)$. Under the above transformations $T_{1}$ and $T_{2}$ take the following 
forms:

$$
\begin{aligned}
T_{1} & =\bar{Y}\left(1+e_{1}\right)-\sum_{j=1}^{p} \beta_{y z j} \bar{Z}_{j} e_{5 j}\left(1+e_{8 j}\right)\left(1+e_{9 j}\right)^{-1} \\
T_{2} & =\left\{\bar{Y}\left(1+e_{2}\right)-\sum_{j=1}^{p} \beta_{y z j} \bar{Z}_{j} e_{6 j}\left(1+e_{10 j}\right)\left(1+e_{11 j}\right)^{-1}\right\} \\
& \times\left\{\left(1+e_{4}\right)-\sum_{j=1}^{p} \frac{\beta_{x z j} \bar{Z}_{j}}{\bar{X}} e_{7 j}\left(1+e_{13 j}\right)\left(1+e_{14 j}\right)^{-1}\right\} \\
& \times\left\{1+\left(e_{3}-\sum_{j=1}^{p} \frac{\beta_{x z j} \bar{Z}_{j}}{\bar{X}} e_{6 j}\left(1+e_{12 j}\right)\left(1+e_{11 j}\right)^{-1}\right)\right\}^{-1}
\end{aligned}
$$

Thus, we have the following theorems:

Theorem 1: Bias of the estimator $T$ to the first order of approximations is obtained as

$$
B(T)=\varphi B\left(T_{1}\right)+(1-\varphi) B\left(T_{2}\right)
$$

where

$$
\begin{aligned}
B\left(T_{1}\right)= & \left(\frac{1}{u}-\frac{1}{N}\right) \sum_{j=1}^{p} \beta_{y z j}\left(\frac{\alpha_{003}}{S_{z j}^{2}}-\frac{\alpha_{012}}{S_{y z j}}\right) \\
B\left(T_{2}\right)= & \left(\frac{1}{m}-\frac{1}{N}\right) \sum_{j=1}^{p} \beta_{y z j}\left(\frac{\alpha_{003}}{S_{z j}^{2}}-\frac{\alpha_{012}}{S_{y z j}}\right)+\left(\frac{1}{m}-\frac{1}{n}\right) \frac{\bar{Y}}{\bar{X}}\left[\left(\frac{S_{x}^{2}}{\bar{X}}-\frac{S_{y x}}{\bar{Y}}\right)\right. \\
& +\sum_{j=1}^{p} \beta_{x z j}\left(\frac{\alpha_{102}}{S_{x z j}}-\frac{\alpha_{003}}{S_{z j}^{2}}\right)+\sum_{j=1}^{p} \beta_{x z j}\left(\frac{S_{y z j}}{\bar{Y}}-\frac{S_{x z j}}{\bar{X}}\right) \\
& \left.+\sum_{j \neq k=1}^{p} \beta_{x z k}\left(\frac{\beta_{x z j}}{\bar{X}}-\frac{\beta_{y z j}}{\bar{Y}}\right) S_{z j z k}\right]
\end{aligned}
$$

where $\alpha_{r s t}=E\left[(x-\bar{X})^{r}(y-\bar{Y})^{s}\left(z_{j}-\bar{Z}_{j}\right)^{t}\right] ;(r, s, t \geq 0)$ are integers and $(j=1,2,3, \ldots, p)$. Proof: The bias of the estimator $T$ is given by

$$
\begin{aligned}
B(T) & =E[T-\bar{Y}]=\varphi E\left(T_{1}-\bar{Y}\right)+(1-\varphi) E\left(T_{2}-\bar{Y}\right) \\
& =\varphi B\left(T_{1}\right)+(1-\varphi) B\left(T_{2}\right)
\end{aligned}
$$


where $B\left(T_{1}\right)=E\left[T_{1}-\bar{Y}\right]$ and $B\left(T_{2}\right)=E\left[T_{2}-\bar{Y}\right]$.

The bias of the estimators $T_{1}$ and $T_{2}$ are derived as follows:

$$
B\left(T_{1}\right)=E\left[T_{1}-\bar{Y}\right]
$$

Substituting the expression of the estimator $T_{1}$ from equation (4) and expanding it binomially, taking expectations and retaining the terms up-to the first order of approximations, we have the bias of the estimator $T_{1}$ as shown in equation (7). Similarly, with the help of expression (5) we get the bias of the estimator $T_{2}$ as shown in equation (8). Now substituting the values of $B\left(T_{1}\right)$ and $B\left(T_{2}\right)$ in the equation (9), we get the bias of the estimator $T$ as shown in equation (6).

Theorem 2: Mean square error of the estimator $T$ to the first order of approximations is obtained as

$$
M(T)=\varphi^{2} M\left(T_{1}\right)+(1-\varphi)^{2} M\left(T_{2}\right)+2 \varphi(1-\varphi) C\left(T_{1}, T_{2}\right)
$$

where

$$
\begin{aligned}
M\left(T_{1}\right)= & \left(\frac{1}{u}-\frac{1}{N}\right)\left(1-\sum_{j=1}^{p} \rho_{y z j}^{2}+\sum_{j \neq k=1}^{p} \rho_{y z j} \rho_{y z k} \rho_{z j z k}\right) S_{y}^{2} \\
M\left(T_{2}\right)= & {\left[\left(\frac{1}{m}-\frac{1}{N}\right)\left(1-\sum_{j=1}^{p} \rho_{y z j}^{2}+\sum_{j \neq k=1}^{p} \rho_{y z j} \rho_{y z k} \rho_{z j z k}\right)+\left(\frac{1}{m}-\frac{1}{n}\right)\right.} \\
& \left.\times\left\{1-2 \rho_{y x}+\sum_{j=1}^{p} \rho_{y z j}^{2}-\sum_{j \neq k=1}^{p} \rho_{y z j} \rho_{y z k} \rho_{z j z k}\right\}\right] S_{y}^{2} \\
C\left(T_{1}, T_{2}\right)= & -\frac{1}{N}\left(1-\sum_{j=1}^{p} \rho_{y z j}^{2}+\sum_{j \neq k=1}^{p} \rho_{y z j} \rho_{y z k} \rho_{z j z k}\right) S_{y}^{2}
\end{aligned}
$$

Proof: It is obvious that mean square error of the estimator $T$ is given by

$$
\begin{aligned}
M(T) & =E[T-\bar{Y}]^{2}=E\left[\varphi\left(T_{1}-\bar{Y}\right)+(1-\varphi)\left(T_{2}-\bar{Y}\right)\right]^{2} \\
& =\varphi^{2} M\left(T_{1}\right)+(1-\varphi)^{2} M\left(T_{2}\right)+2 \varphi(1-\varphi) C\left(T_{1}, T_{2}\right)
\end{aligned}
$$

where $M\left(T_{1}\right)=E\left[T_{1}-\bar{Y}\right]^{2}, M\left(T_{2}\right)=E\left[T_{2}-\bar{Y}\right]^{2}$ and $C\left(T_{1}, T_{2}\right)=E\left[\left(T_{1}-\bar{Y}\right)\left(T_{2}-\bar{Y}\right)\right]$

The mean square errors of the estimators $T_{1}$ and $T_{2}$ are derived as follows:

$$
M\left(T_{1}\right)=E\left[T_{1}-\bar{Y}\right]^{2}
$$

Substituting the expression of the estimator $T_{1}$ from equation (4) and expanding it binomially, taking expectations and retaining the terms up-to the first order of approximations, we have the expression of $M\left(T_{1}\right)$ as shown in equation (11). 
Similarly, with the help of expression (5) we get

$$
\begin{aligned}
M\left(T_{2}\right)= & {\left[\left(\frac{1}{m}-\frac{1}{N}\right)\left(1-\sum_{j=1}^{p} \rho_{y z j}^{2}+\sum_{j \neq k=1}^{p} \rho_{y z j} \rho_{y z k} \rho_{z j z k}\right)+\left(\frac{1}{m}-\frac{1}{n}\right)\right.} \\
& \times\left\{1-2 \rho_{y x}-\sum_{j=1}^{p} \rho_{x z j}^{2}+2 \sum_{j=1}^{p} \rho_{x z j} \rho_{y z j}+\sum_{j \neq k=1}^{p} \rho_{x z j} \rho_{x z k} \rho_{z j z k}\right. \\
& \left.\left.-2 \sum_{j \neq k=1}^{p} \rho_{x z j} \rho_{y z k} \rho_{z j z k}\right\}\right] S_{y}^{2}
\end{aligned}
$$

Further, we consider $\rho_{x z j}=\rho_{y z j},(j=1,2, \ldots, p)$, which is an intuitive assumption, considered by Cochran [8] and Feng and Zou [11]. In the light of this assumption the above equation of $M\left(T_{2}\right)$ takes the form as shown in equation (12).

The covariance type term between the estimators $T_{1}$ and $T_{2}$ is derived as

$$
C\left(T_{1}, T_{2}\right)=E\left[\left(T_{1}-\bar{Y}\right)\left(T_{2}-\bar{Y}\right)\right]
$$

Substituting the expressions of $T_{1}$ and $T_{2}$ from the equations (4) and (5), expanding binomially, taking expectations and retaining the terms up-to order $n^{-1}$, we have the expression of $C\left(T_{1}, T_{2}\right)$ as shown in equation (13). Now substituting the values of $M\left(T_{1}\right), M\left(T_{2}\right)$ and $C\left(T_{1}, T_{2}\right)$ in the equation (14), we get the mean square error of the estimator $T$ as it is given in equation (10).

Remark 1: Results shown in equations (11) - (13) are derived under the assumption that the coefficients of variation of $x, y$ and $z$ are approximately equal.

\section{Minimum Mean Square Error of the Estimator $T$}

Since, the mean square error of the estimator $T$ in equation (10) is a function of unknown constant $\varphi$, therefore, it is minimized with respect to $\varphi$ and subsequently the optimum value of $\varphi$ is obtained as

$$
\varphi_{o p t}=\frac{M\left(T_{2}\right)-C\left(T_{1}, T_{2}\right)}{M\left(T_{1}\right)+M\left(T_{2}\right)-2 C\left(T_{1}, T_{2}\right)}
$$


Now substituting the value of $\varphi_{\text {opt }}$ from equation (15) in equation (10), we get the optimum mean square error of the estimator $T$ as

$$
M(T)_{o p t}=\frac{M\left(T_{1}\right) \cdot M\left(T_{2}\right)-\left\{C\left(T_{1}, T_{2}\right)\right\}^{2}}{M\left(T_{1}\right)+M\left(T_{2}\right)-2 C\left(T_{1}, T_{2}\right)}
$$

Further, substituting the values from equations (11) - (13) in equations (15) and (16), we get the simplified values of $\varphi_{o p t}$ and $M(T)_{o p t}$ as:

$$
\begin{aligned}
\varphi_{o p t} & =\frac{\mu\left[A_{1}+\mu A_{2}\right]}{A_{1}+\mu^{2} A_{2}} \\
M(T)_{\text {opt }} & =\frac{A_{1}}{n}\left[\frac{A_{1}+\mu A_{2}}{A_{1}+\mu^{2} A_{2}}-f\right] S_{y}^{2}
\end{aligned}
$$

where $A_{1}=1-\sum_{j=1}^{p} \rho_{y z j}^{2}+\sum_{j \neq k=1}^{p} \rho_{y z j} \rho_{y z k} \rho_{z j z k}$, $A_{2}=1-2 \rho_{y x}+\sum_{j=1}^{p} \rho_{y z j}^{2}-\sum_{j \neq k=1}^{p} \rho_{y z j} \rho_{y z k} \rho_{z j z k}, f=\frac{n}{N}$ and $\mu$ is the fraction of the fresh sample drawn on the current (second) occasion corresponding to the estimator $T$.

\section{Optimum Replacement Policy}

To determine the optimum value of $\mu$ so that population mean $\bar{Y}$ may be estimated with the maximum precision, we minimize $M(T)_{\text {opt }}$ in equation (18) with respect to $\mu$, which result in quadratic equation in $\mu$, which is shown as

$$
A_{2} \mu^{2}+2 A_{1} \mu-A_{1}=0
$$

Solving equation (19) for $\mu$, the solutions are given as

$$
\mu_{\text {opt }}=\frac{-A_{1} \pm \sqrt{A_{1}^{2}+A_{1} A_{2}}}{A_{2}}
$$

The real values of $\mu_{\text {opt }}$ exist, if $\left(A_{1}^{2}+A_{1} A_{2}\right) \geq 0$. For any combinations of correlations, which satisfies this condition, two real values of $\mu_{\text {opt }}$ are possible, hence, to choose a value of $\mu_{o p t}$, it should be remembered that $0 \leq \hat{\mu} \leq 1$, all other values of $\mu_{\text {opt }}$ are inadmissible. If both the values are admissible, the lower one is the best choice. Substituting the admissible values of $\mu_{\text {opt }}$ say $\mu_{0}$ from equation (20) in equation (18), we have the 
optimum value of the mean square error of the estimator $T$, which is shown as

$$
M\left(T^{0}\right)_{o p t}=\frac{A_{1}}{n}\left[\frac{A_{1}+\mu_{0} A_{2}}{A_{1}+\mu_{0}^{2} A_{2}}-f\right] S_{y}^{2}
$$

\section{Special Case}

There may be several instances where the $p$-auxiliary variables are mutually uncorrelated but they are correlated to study variable. For example, in a survey of commercial products say the aim is to estimate the number of persons reading newspaper, then in that case the number of copies produced by different newspapers are different and the number of copies produced by a particular newspaper company is uncorrelated to the number of copies produced by another newspaper but both are correlated to the study variable, i.e., number of persons reading newspaper. Hence, for modeling such type of situations in the proposed estimator, we consider the case where the $p$-auxiliary variables are mutually uncorrelated, i.e., $\rho_{z j z k}=0 \quad \forall j \neq k=1,2, \ldots, p$ then the expression for the optimum value of $\mu$ and $M\left(T^{0}\right)_{\text {opt }}$ reduces to

$$
\mu_{o p t}=\frac{-A_{1}^{*} \pm \sqrt{A_{1}^{* 2}+A_{1}^{*} A_{2}^{*}}}{A_{2}^{*}}
$$

and

$$
M\left(T^{0}\right)_{o p t}=\frac{A_{1}^{*}}{n}\left[\frac{A_{1}^{*}+\mu_{0} A_{2}^{*}}{A_{1}^{*}+\mu_{0}^{2} A_{2}^{*}}-f\right] S_{y}^{2}
$$

where

$$
A_{1}^{*}=1-\sum_{j=1}^{p} \rho_{y z j}^{2} \quad \text { and } \quad A_{2}^{*}=1-2 \rho_{y x}+\sum_{j=1}^{p} \rho_{y z j}^{2}
$$

\section{Efficiency Comparison}

The percent relative efficiencies of $T$ with respect to (i) sample mean $\bar{y}_{n}$, when there is no matching and (ii) $\hat{\bar{Y}}=\varphi^{*} \bar{y}_{u}+\left(1-\varphi^{*}\right) \bar{y}_{m}^{\prime}$, when no auxiliary information is used at any occasion, where $\bar{y}_{m}^{\prime}=\bar{y}_{m}+\beta_{y x}\left(\bar{x}_{n}-\bar{x}_{m}\right)$, have 
been obtained for different choices of the correlations involved. Variance of the estimator $\bar{y}_{n}$ and the optimum variance of the estimator $\hat{\bar{Y}}$ are given by

$$
\begin{aligned}
V\left(\bar{y}_{n}\right) & =\frac{1}{n}(1-f) S_{y}^{2} \\
V(\hat{\bar{Y}})_{o p t^{*}} & =\frac{1}{2 n}\left[1+\sqrt{\left(1-\rho_{y x}^{2}\right)}-2 f\right] S_{y}^{2}
\end{aligned}
$$

The percent relative efficiencies $E_{1}$ and $E_{2}$ of $T$ (under optimal condition) with respect to $\bar{y}_{n}$ and $\hat{\bar{Y}}$ respectively are given by

$$
E_{1}=\frac{V\left(\bar{y}_{n}\right)}{M\left(T^{0}\right)_{o p t}} \times 100 \text { and } E_{2}=\frac{V(\hat{\bar{Y}})_{o p t^{*}}}{M\left(T^{0}\right)_{o p t}} \times 100
$$

\section{Empirical Study}

The expressions of the optimum $\mu\left(\right.$ i.e. $\left.\mu_{0}\right)$ and the percent relative efficiencies $E_{1}$ and $E_{2}$ are in terms of population correlation coefficients. Therefore, the values of $\mu_{0}, E_{1}$ and $E_{2}$ have been computed for different choices of positive correlations while the value of $f$ (sampling fraction) is chosen as 0.1. For empirical studies, cases of $p=1,2$ and 3 have been considered.

Case 1: For $p=1$, the values of $A_{1}$ and $A_{2}$ take the form $A_{1}=1-\rho_{y z}^{2}$ and $A_{2}=1-2 \rho_{y x}+\rho_{y z}^{2}$, which is the work of Singh and Karna [21].

Case 2: For $p=2$ and assuming that the two auxiliary variables are correlated i.e., $\rho_{z 1 z 2} \neq 0$. The values of $A_{1}$ and $A_{2}$ are given by $A_{1}=$ $1-\left(\rho_{y z 1}^{2}+\rho_{y z 2}^{2}\right)+2 \rho_{y z 1} \rho_{y z 2} \rho_{z 1 z 2}$ and $A_{2}=1-2 \rho_{y x}+\left(\rho_{y z 1}^{2}+\rho_{y z 2}^{2}\right)-$ $2 \rho_{y z 1} \rho_{y z 2} \rho_{z 1 z 2}$. Substituting these values of $A_{1}$ and $A_{2}$ in equations (20) and (21), we have the values of optimum $\mu, M\left(T^{0}\right)_{\text {opt }}, E_{1}$ and $E_{2}$. For different choices of correlations, Tables $1-2$ show the optimum values of $\mu$ i.e., $\mu_{0}$ and percent relative efficiencies $E_{1}$ and $E_{2}$ of the estimator $T$ (under optimal condition) with respect to $\bar{y}_{n}$ and $\hat{\bar{Y}}$ respectively.

Case 3: For $p=2$ and assuming that the two auxiliary variables are uncorrelated i.e., $\rho_{z 1 z 2}=0$. The values of $A_{1}^{*}$ and $A_{2}^{*}$ are given by $A_{1}^{*}=$ $1-\left(\rho_{y z 1}^{2}+\rho_{y z 2}^{2}\right)$ and $A_{2}^{*}=1-2 \rho_{y x}+\left(\rho_{y z 1}^{2}+\rho_{y z 2}^{2}\right)$. Using these values in equations (20) and (21), the optimum values of $\mu, E_{1}$ and $E_{2}$ are shown in Table 3 .

Case 4: For $p=3$ and assuming that the two auxiliary variables are correlated i.e., $\rho_{z 1 z 2} \neq 0 \quad \forall j \neq k=1,2$,3. In this case the values of $A_{1}$ and $A_{2}$ take the following form: 
Table 1: Optimum values of $\mu$ and percent relative efficiencies of $T$ with respect to $\bar{y}_{n}$ and $\hat{\bar{Y}}$ for $\rho_{y x}=0.7$.

\begin{tabular}{|c|c|c|c|c|c|c|c|c|c|c|}
\hline \multicolumn{1}{c}{$\rho_{y z 1}$} & \multicolumn{1}{c}{0.5} & \multicolumn{1}{c}{0.7} & \multicolumn{4}{c|}{0.9} \\
\hline$\rho_{y z 2}$ & $\rho_{z 1 z 2}$ & $\mu_{0}$ & $E_{1}$ & $E_{2}$ & $\mu_{0}$ & $E_{1}$ & $E_{2}$ & $\mu_{0}$ & $E_{1}$ & $E_{2}$ \\
\hline 0.5 & 0.5 & 0.527 & 141.6 & 119.1 & 0.502 & 164.6 & 138.5 & 0.446 & 226.2 & 190.2 \\
& 0.7 & 0.543 & 129.1 & 108.6 & 0.527 & 141.6 & 119.1 & 0.493 & 172.9 & 145.4 \\
& 0.9 & 0.557 & 118.8 & $* *$ & 0.549 & 124.7 & 104.9 & 0.527 & 141.6 & 119.1 \\
\hline 0.7 & 0.5 & 0.502 & 164.6 & 138.5 & 0.479 & 187.2 & 157.5 & 0.425 & 253.8 & 213.5 \\
& 0.7 & 0.527 & 141.6 & 119.1 & 0.520 & 148.0 & 124.5 & 0.496 & 170.3 & 143.3 \\
& 0.9 & 0.549 & 124.7 & 104.9 & 0.550 & 123.5 & 103.9 & 0.541 & 130.9 & 110.1 \\
\hline 0.9 & 0.5 & 0.446 & 226.2 & 190.2 & 0.425 & 253.8 & 213.5 & 0.360 & 367.6 & 309.2 \\
& 0.7 & 0.493 & 172.9 & 145.4 & 0.496 & 170.3 & 143.3 & 0.480 & 186.2 & 156.6 \\
& 0.9 & 0.527 & 141.6 & 119.1 & 0.541 & 130.9 & 110.1 & 0.541 & 130.4 & 109.7 \\
\hline
\end{tabular}

Note: "**" indicates no gain.

Table 2: Optimum values of $\mu$ and percent relative efficiencies of $T$ with respect to $\bar{y}_{n}$ and $\hat{\bar{Y}}$ for $\rho_{y x}=0.9$.

\begin{tabular}{|c|c|c|c|c|c|c|c|c|c|c|}
\hline \multicolumn{1}{c}{$\rho_{y z 1}$} & \multicolumn{1}{c}{0.5} & \multicolumn{1}{c|}{0.9} \\
\hline$\rho_{y z 2}$ & $\rho_{z 1 z 2}$ & $\mu_{0}$ & $E_{1}$ & $E_{2}$ & $\mu_{0}$ & $E_{1}$ & $E_{2}$ & $\mu_{0}$ & $E_{1}$ & $E_{2}$ \\
\hline 0.5 & 0.5 & 0.695 & 182.3 & 125.1 & 0.635 & 214.9 & 147.6 & 0.582 & 304.4 & 209.0 \\
& 0.7 & 0.673 & 164.7 & 113.14 & 0.659 & 182.3 & 125.1 & 0.628 & 226.8 & 155.7 \\
& 0.9 & 0.685 & 150.5 & 103.3 & 0.678 & 158.7 & 108.9 & 0.659 & 182.3 & 125.1 \\
\hline 0.7 & 0.5 & 0.635 & 214.9 & 147.6 & 0.614 & 247.4 & 169.9 & 0.562 & 345.5 & 237.2 \\
& 0.7 & 0.659 & 182.3 & 125.1 & 0.652 & 191.3 & 131.3 & 0.630 & 223.1 & 153.1 \\
& 0.9 & 0.678 & 158.7 & 108.9 & 0.679 & 157.0 & 107.8 & 0.671 & 167.3 & 114.9 \\
\hline 0.9 & 0.5 & 0.582 & 304.4 & 209.0 & 0.562 & 345.5 & 237.2 & 0.493 & 518.8 & 356.2 \\
& 0.7 & 0.628 & 226.8 & 155.7 & 0.630 & 223.1 & 153.1 & 0.615 & 245.9 & 168.8 \\
& 0.9 & 0.659 & 182.3 & 125.1 & 0.671 & 167.3 & 114.9 & 0.671 & 166.6 & 114.4 \\
\hline
\end{tabular}

Table 3: Optimum values of $\mu$ and percent relative efficiencies of $T$ with respect to $\bar{y}_{n}$ and $\hat{\bar{Y}}$ for $\rho_{z 1 z 2}=0$.

\begin{tabular}{c|c|c|c|c|c|c|c|c|c|c|}
\hline \multicolumn{10}{c}{0.5} & \multicolumn{1}{c}{0.7} \\
\hline$\rho_{y z 1}$ & $\rho_{y z 2}$ & $\mu_{0}$ & $E_{1}$ & $E_{2}$ & $\mu_{0}$ & $E_{1}$ & $E_{2}$ & $\mu_{0}$ & $E_{1}$ & $E_{2}$ \\
\hline 0.3 & 0.3 & 0.475 & 115.2 & 106.6 & 0.539 & 132.6 & 111.5 & 0.669 & 169.6 & 116.4 \\
& 0.5 & 0.448 & 134.2 & 124.2 & 0.511 & 155.5 & 130.8 & 0.645 & 201.9 & 138.6 \\
& 0.7 & 0.393 & 182.9 & 169.2 & 0.455 & 214.7 & 180.6 & 0.591 & 287.6 & 197.4 \\
\hline 0.5 & 0.3 & 0.448 & 134.2 & 124.2 & 0.511 & 155.5 & 130.8 & 0.645 & 201.9 & 138.6 \\
& 0.5 & 0.414 & 162.5 & 150.4 & 0.477 & 189.9 & 159.7 & 0.612 & 251.3 & 172.5 \\
& 0.7 & 0.337 & 250.7 & 232.0 & 0.397 & 298.5 & 251.1 & 0.532 & 412.8 & 283.4 \\
\hline 0.7 & 0.3 & 0.393 & 182.9 & 169.2 & 0.455 & 214.7 & 180.6 & 0.591 & 287.6 & 197.4 \\
& 0.5 & 0.337 & 250.7 & 232.0 & 0.397 & 298.5 & 251.1 & 0.532 & 412.8 & 283.4 \\
& 0.7 & 0.123 & 1143 & 1058 & 0.154 & 1433 & 1206 & 0.240 & 2271 & 1559 \\
\hline
\end{tabular}


$A_{1}=1-\left(\rho_{y z 1}^{2}+\rho_{y z 2}^{2}+\rho_{y z 3}^{2}\right)+2\left(\rho_{y z 1} \rho_{y z 2} \rho_{z 1 z 2}+\rho_{y z 1} \rho_{y z 3} \rho_{z 1 z 3}+\rho_{y z 2} \rho_{y z 3} \rho_{z 2 z 3}\right)$ and $A_{2}=$ $1-2 \rho_{y x}+\left(\rho_{y z 1}^{2}+\rho_{y z 2}^{2}+\rho_{y z 3}^{2}\right)-2\left(\rho_{y z 1} \rho_{y z 2} \rho_{z 1 z 2}+\rho_{y z 1} \rho_{y z 3} \rho_{z 1 z 3}+\rho_{y z 2} \rho_{y z 3} \rho_{z 2 z 3}\right)$.

In this case there are seven different correlations. For few sets of these seven correlations optimum values of $\mu$ i.e., $\mu_{0}$ and percent relative efficiencies $E_{1}$ and $E_{2}$ of the estimator $T$ (under optimal condition) with respect to $\bar{y}_{n}$ and $\hat{\bar{Y}}$ respectively have been computed and shown below:

Set1: $\rho_{y x}=0.3, \rho_{y z 1}=0.9, \rho_{y z 2}=0.9, \rho_{y z 3}=0.9, \rho_{z 1 z 2}=0.3, \rho_{z 2 z 3}=0.3$, $\rho_{z 1 z 3}=0.3, \mu_{0}=0.1239, E_{1}=816.7343, E_{2}=795.8347$

Set2: $\rho_{y x}=0.5, \rho_{y z 1}=0.9, \rho_{y z 2}=0.9, \rho_{y z 3}=0.9, \rho_{z 1 z 2}=0.3, \rho_{z 2 z 3}=0.3$, $\rho_{z 1 z 3}=0.3, \mu_{0}=0.1433, E_{1}=948.7066, E_{2}=878.0940$

Set3: $\rho_{y x}=0.7, \rho_{y z 1}=0.9, \rho_{y z 2}=0.9, \rho_{y z 3}=0.9, \rho_{z 1 z 2}=0.3, \rho_{z 2 z 3}=0.3$, $\rho_{z 1 z 3}=0.3, \mu_{0}=0.1776, E_{1}=1184.1, E_{2}=996.0495$

Set4: $\rho_{y x}=0.9, \rho_{y z 1}=0.9, \rho_{y z 2}=0.9, \rho_{y z 3}=0.9, \rho_{z 1 z 2}=0.3, \rho_{z 2 z 3}=0.3$, $\rho_{z 1 z 3}=0.3, \mu_{0}=0.2723, E_{1}=1851.2, E_{2}=1271.1$

Case 5: For $p=3$ and assuming that the two auxiliary variables are independent (uncorrelated) i.e., $\rho_{z j z k}=0 \forall j \neq k=1,2$, 3. In this case the values of $A_{1}$ and $A_{2}$ take the following form: $A_{1}=1-\left(\rho_{y z 1}^{2}+\rho_{y z 2}^{2}+\rho_{y z 3}^{2}\right)$ and $A_{2}=1-2 \rho_{y x}+\left(\rho_{y z 1}^{2}+\rho_{y z 2}^{2}+\rho_{y z 3}^{2}\right)$ For few sets of above four correlations, the values of $\mu_{0}, E_{1}$ and $E_{2}$ are shown below:

Set1: $\rho_{y x}=0.3, \rho_{y z 1}=0.3, \rho_{y z 2}=0.5, \rho_{y z 3}=0.7$

$\mu_{0}=0.2584, E_{1}=288.5298, E_{2}=281.1465$

Set2: $\rho_{y x}=0.3, \rho_{y z 1}=0.5, \rho_{y z 2}=0.5, \rho_{y z 3}=0.7$

$\mu_{0}=0.0779, E_{1}=1424.9, E_{2}=1388.5$

Set3: $\rho_{y x}=0.5, \rho_{y z 1}=0.5, \rho_{y z 2}=0.5, \rho_{y z 3}=0.7$

$\mu_{0}=0.0909, E_{1}=1666.7, E_{2}=1542.6$

Set4: $\rho_{y x}=0.7, \rho_{y z 1}=0.7, \rho_{y z 2}=0.7, \rho_{y z 3}=0.5$

$\mu_{0}=0.1143, E_{1}=2106.3, E_{2}=1771.8$

Set5: $\rho_{y x}=0.9, \rho_{y z 1}=0.5, \rho_{y z 2}=0.5, \rho_{y z 3}=0.7$

$\mu_{0}=0.1827, E_{1}=3414.2, E_{2}=2344.2$

\section{Conclusion}

The following conclusions can be read out from the empirical studies:

(1) From Tables $1-2$ it is vindicated that

(a) For the fixed values of $\rho_{z 1 z 2}, \rho_{y z 1}$, and $\rho_{y z 2}$, the values of $\mu_{0}, E_{1}$ and $E_{2}$ increase with the increasing values of $\rho_{y x}$. This behavior is in agreement 
with the Sukhatme et al. [22] results, which explains that the more the value of $\rho_{y x}$, more the fraction of fresh sample is required on the current occasion.

(b) For the fixed values of $\rho_{y x}, \rho_{z 1 z 2}$ and $\rho_{y z 1}$, the values of $\mu_{0}$ decrease and $E_{1}$ and $E_{2}$ increase with the increasing values of $\rho_{y z 2}$. Similarly, for fixed values of $\rho_{y x}, \rho_{z 1 z 2}$ and $\rho_{y z 2}$, the optimum values of $\mu_{0}$ decrease and $E_{1}$ and $E_{2}$ increase with the increasing values of $\rho_{y z 1}$. These patterns indicate that smaller fresh sample on the current occasion is required, if highly correlated auxiliary variables are available.

(c) For the fixed values of $\rho_{y x}, \rho_{y z 1}$ and $\rho_{y z 2}$, the values of $\mu_{0}$ increase with the increasing values of $\rho_{z 1 z 2}$ while $E_{1}$ and $E_{2}$ are decreasing with increasing trends in $\rho_{z 1 z 2}$, it means that auxiliary variables are quite sensitive with respect to the relation between them.

(2) From Table 3, when the auxiliary variables are uncorrelated, it can be observed that

(a) For the fixed values of $\rho_{y z 1}$ and $\rho_{y z 2}$ the values of $\mu_{0}, E_{1}$ and $E_{2}$ increase with the increasing values of $\rho_{y x}$, which is a desirable result.

(b) For fixed values of $\rho_{y z 1}$ and $\rho_{y x}$ the values of $\mu_{0}$ decrease, while $E_{1}$ and $E_{2}$ increase with the increasing values of $\rho_{y z 2}$. Similar patterns are visible for the case when the values of $\rho_{y z 2}$ and $\rho_{y x}$ are fixed and increasing values of $\rho_{y z 1}$ are observed.

(3) For $p=3$ and when the three auxiliary variables are mutually correlated, we observed that no specific pattern is seen as for so many combinations of correlations the optimum values of $\mu$ do not exist. This behavior suggests that the correlation between the auxiliary variable do not play significant role in terms of proposed estimator.

(4) For $p=3$ and when the three auxiliary variables are uncorrelated then for fixed values of $\rho_{y x}, \rho_{z 1 z 3}, \rho_{z 2 z 3}, \rho_{z 1 z 2}, \rho_{y z 2}$ and $\rho_{y z 3}$ the values of $\mu_{0}$ decrease, while $E_{1}$ and $E_{2}$ increase with the increasing values of $\rho_{y z 1}$. Similar patterns are observed if the case for the increasing values of $\rho_{y z 2}$ or $\rho_{y z 3}$ is taken into account.

(5) The results obtained for $p=1$ and $p=2$ are quite appreciable, while when the number of auxiliary variables increase, the expressions become complex due to increase in the number of correlations, hence, practically, it is more realistic to use two auxiliary variables out of several available auxiliary variables. 
Thus it is clear that the use of the auxiliary variables are highly rewarding in terms of the proposed estimator. It is also clear that if highly correlated auxiliary variables are used, relatively only a smaller fraction of sample on the current (second) occasion is desired to be replaced by a fresh sample which reduces the cost of the survey. Hence it can be recommended for future use.

\section{Acknowledgement}

Authors are thankful to the referees for their valuable and inspiring suggestions. Authors are also thankful to UGC, New Delhi and the Indian School of Mines, Dhanbad for providing financial assistances to carry out the present research work.

\section{References}

1. R. J. Jessen. Statistical investigation of a sample survey for obtaining farm facts. Iowa Agricultural Experiment Station Research Bulletin, 304:1-104, 1942.

2. H. D. Patterson. Sampling on successive occasions with partial replacement of units. Journal of the Royal Statistical Society, 12:241-255, 1950.

3. A. R. Eckler. Rotation sampling. Ann. Math. Stat., 26:664-685, 1955.

4. J. N. K. Rao and J. E. Graham. Rotation design for sampling on repeated occasions. Jour. Amer. Statist. Assoc., 59:492-509, 1964.

5. A. R. Sen. Successive sampling with two auxiliary variables. Sankhya, 33(B):371-378, 1971.

6. A. R. Sen. Successive sampling with $\mathrm{p}(p \geq 1)$ auxiliary variables. Ann. Math. Statist., 43:2031-2034, 1972.

7. A. R. Sen. Theory and application of sampling on repeated occasions with several auxiliary variables. Biometrics, 29:381-385, 1973.

8. W. G. Cochran. Sampling Techniques. New York: John Wiley and Sons, 1977. 
9. P. C. Gupta. Sampling on two successive occasions. Jour. Statist. Res., 13:7-16, 1979.

10. V. K. Singh, G. N. Singh, and D. Shukla. An efficient family of ratiocum-difference type estimators in successive sampling over two occasions. J. Sci. Res., 41(C):149-159, 1991.

11. S. Feng and G. Zou. Sample rotation method with auxiliary variable. Communications in Statistics: Theory and Methods, 26(6):1497-1509, 1997.

12. R. S. Biradar and H. P. Singh. Successive sampling using auxiliary information on both occasions. Cal. Statist. Assoc. Bull, (51):243-251, 2001.

13. G. N. Singh and V. K. Singh. On the use of auxiliary information in successive sampling. J. Indian Soc. Agric. Statist., 54(1):1-12, 2001.

14. G. N. Singh. Estimation of population mean using auxiliary information on recent occasion in h-occasion successive sampling. Statistics in Transition, 6:523-532, 2003.

15. G. N. Singh. On the use of chain-type ratio estimator in successive sampling. Statistics in Transition, 7:21-26, 2005.

16. G. N. Singh and K. Priyanka. On the use of chain-type ratio to difference estimator in successive sampling. IJAMAS, 5(S06):41-49, 2006.

17. G. N. Singh and K. Priyanka. On the use of auxiliary information in search of good rotation patterns on successive occasions. Bulletin of Statistics and Economics, 1(A07)):42-60, 2007.

18. G. N. Singh and K. Priyanka. Search of good rotation patterns to improve the precision of estimates at current occasion. Communications in Statistics- Theory and Methods, 37(3):337-348, 2008.

19. G. N. Singh and K. Priyanka. On the use of several auxiliary variates to improve the precision of estimates at current occasion estimation of population mean at current occasion in presence of several varying auxiliary variates in two-occasion successive sampling. J. Indian Soc. Agric. Statist., 62(3):253-265, 2008. 
20. G. N. Singh and J. P. Karna. Estimation of population mean on current occasion in two-occasion successive sampling. METRON, 67(1):69-85, 2009 .

21. G. N. Singh and J. P. Karna. Search of effective rotation patterns in presence of auxiliary information in successive sample over twooccasions. Statistics in Transition-new series, 10(1):59-73, 2009.

22. P. V. Sukhatme, B. V. Sukhatme, S. Sukhatme, and C. Asok. Sampling theory of surveys with applications. Iowa State University Press, Ames, Iowa (USA) and Indian Society of Agricultural Statistics, New Delhi (India), 1984. 